\title{
Liver transplantation in the most severely ill cirrhotic patients
}

\author{
Obed A*, Bashir A and Jarrad A \\ Department of Hepatobiliary and Liver Transplant, Jordan Hospital, Amman, Jordan
}

Louvet and colleagues are to be congratulated on their work that presents a significant survival rate benefit in transplanted patients suffering from ACLF-3 compered with non-transplanted controls [1].

There remain a few points that should be highlighted.

In 2011 we published an observational study where patients with ACLF-3 remained on the waiting list despite suffering from active pneumonia. Of the initially nine included patients only six patients improved under our proposed goal-directed therapy and subsequently underwent liver transplantation (LT). Before the development of pneumonia, the patients must have mild symptoms of chronic liver failure, corresponding to Child-Pugh-Turcotte class A with a median MELD of 12. All patients subsequently developed severe liver dysfunction that fulfilled the criteria for ACLF-3 [2].

After liver transplantation all six patients recovered quickly from infection and multiorgan failure; only one patient died because of late complications (CMV colitis). We proposed that in patients with ACLF and active pneumonia transplantation is a treatment option that should not hastily be abandoned, if patients clinically respond and stabilize.

Secondly, Louvet et al. impressively have shown that LT can improve the very poor prognosis of the most severely ill patients with cirrhosis (ACLF-3), allowing them to reach survival rates comparable to transplanted patients with a lower ACLF score. Furthermore, Louvet et al. matched the 73 cases transplanted with ACLF-3 to 119 nontransplanted controls with cirrhosis and multiple organ dysfunction, hospitalized in the ICU. The 1-year survival rate was significantly higher in transplanted patients than in controls ( $83.6 \%$ vs. $7.9 \%)$.

We agree with Louvet et al. that BAR and P-SOFT scores appeared to overestimate the risk of death in patients with ACLF-3. Based on these two scores only, many patients would be disqualified for LT ("futile LT"). Analogous to our findings Louvet et al. also considered the "transplantation window" as fundamental after the clinical status is stabilized.

These good survival results are associated with an increased postLT hospital stay and elevated percentage of complications. However, to obtain these good results a short decision-making procedure is required due to the short "transplantation window" suggesting that patients with ACLF-3 must be rapidly referred to a specific liver ICU.

We also support very short selection processes in order to reduce the risks of complications and the deterioration of multiorgan failures causing potential preventable death and reducing the probabilities of a patient being transplanted just in time [3].

In spite of the retrospective study design, these clear and significant results should be implemented in therapy recommendations for the clinical routine.

\section{Conflicts of interest}

The authors declared that they do not have anything to disclose regarding funding or conflict of interest with respect to this manuscript.

\section{References}

1. Artru F, louvet A, Ruiz I, Levesque E, Labreuche JJ, et al. (2017) Liver transplantation in the most severely ill cirrhotic patients: A multicenter study in acute-on-chronic liver failure grade $3 . J$ Hepatol 67: 708-715.

2. Goralczyk AD, Ajaj AW, Tsui TY, Bauer M, Lorf T, et al. (2011) Liver transplantation in patients with liver cirrhosis and active pneumonia: an observational study. Transpl Int 24: 1068-1074.

3. Putignano A, Gustot $\mathrm{T}$ (2017) New concepts in acute-on-chronic liver failure: implications for liver transplantation. Liver Transplant 23: 234-243
Copyright: (C2017 Obed A. This is an open-access article distributed under the terms of the Creative Commons Attribution License, which permits unrestricted use, distribution, and reproduction in any medium, provided the original author and source are credited.
Correspondence to: Aiman Obed, MD FACS, FEBBS, Hepatobiliary and Liver Transplant Unit, Jordan Hospital, PO Box 520248, Amman, Jordan, E-mail: aimanobed@hotmail.com

Received: October 11, 2017; Accepted: November 01, 2017; Published: November 03, 2017 